patients with a diagnosed STD. EPT is legal for Chlamydia trachomatis (CT) infections in New York State. To guide EPT implementation at New York City STD (NYC STD) clinics, we estimated potential EPT use and EPT treatments to dispense.

Methods We analysed electronic medical record data for heterosexual patient visits to NYC STD clinics in 2009. To estimate potential EPT use, we measured: proportion clinic patients with presumptive diagnosis of mucopurulent cervicitis (MPC); nonspecific urethritis (NGU); contacts of both who tested positive for CT; patients with laboratory-confirmed genital CT infection; and proportion treated on day of visit. To guide policy on EPT treatments to dispense, we measured the median number of sex partners reported by CT-infected persons in the previous 3 months. To determine whether to route CT contacts to a physician (MD) visit (full STD evaluation) or an express visit (EV), we assessed STD diagnoses among CT contacts.

Results Among clinic patients with presumptive diagnoses of MPC, NGU, and contacts of both, CT prevalence was MPC, 14\% (293/ 2144); MPC contact, 15\% (38/257); NGU, 23\% (1553/6744); and NGU contact, 17\% (113/677). Of 40099 patients tested for CT, 13\% (5402/40 099) had a laboratory-confirmed CT infection. Of those, $79 \%$ (4288/5402) had been treated presumptively on day of visit. Males $(n=4551)$ and females $(n=3186)$ reported a median of two and one sex partners, respectively. Of 3561 contacts with CT diagnosis, $2339(66 \%)$ were asymptomatic on day of visit and were routed to EV. Of those, $936(40 \%)$ had $>1$ diagnosis other than CT; $22 \%$ of those (205) had a diagnosis of herpes simplex virus, human papillomavirus, trichomoniasis, or bacterial vaginosis.

Conclusion EPT is recommended only for heterosexual patients with laboratory-confirmed CT diagnosis because CT-prevalence was low among patients presumptively diagnosed with either MPC or NGU and their contacts. Approximately 20\% of CT-infected persons qualify for EPT; the majority of CT-infected persons are treated on day of visit. EPT-eligible patients should be offered up to three treatments for sex partners. Asymptomatic CT contacts reporting they have taken EPT should be routed to EV; those who report not taking EPT should be routed to an $\mathrm{MD}$ visit regardless of symptoms. Symptomatic CT contacts should receive an MD visit.

\section{P3-S1.11 PER CENT ADDITIONAL TEST POSITIVE FOLLOWING POSITIVE COMBO 2 CHLAMYDIA (CT) AND GONORRHOEA (GC) SPECIMENS: ASSESSING THE IMPACT OF PREVALENCE}

doi:10.1136/sextrans-2011-050108.411

${ }^{1} \mathrm{R}$ Johnson, ${ }^{2} \mathrm{D}$ Ware, ${ }^{3} \mathrm{~L}$ Mena, ${ }^{1} \mathrm{~F}$ Xu. ${ }^{1}$ Centers for Disease Control and Prevention, Atlanta, USA; ${ }^{2}$ Public Health Laboratory, Mississippi, USA; ${ }^{3}$ University of Mississippi, Medical Center, USA

Background The positive predictive value (PPV) of a screening test (ST) is a function of prevalence and ST specificity and is expected to decrease with decreasing prevalence unless ST specificity approaches $100 \%$. Consequently, an additional test (AT) following positive STs may be indicated if prevalence is low. Our objective was to determine the impact of CT and GC prevalence on per cent AT positive following positive STs by Gen-Probe Combo 2 CT and GC using data from public clinics in the state of Mississippi.

Methods Based-on CDC's electronic prevalence monitoring databases from 2005 to 2007, we stratified 126 clinics (with >400 females tested) served by Mississippi State Public Health Laboratory (MSPHL) based on ST positivity. We calculated the per cent AT positive among 6553 CT ST positive and 1841 GC ST positive specimens. We further examined the impact of the quantitative Combo 2 GC results (relative light units $(\mathrm{RLU})$ ) for a sample of 508 specimens from clinics with low $(<2.0 \%$, family planning) and high $(>6.0 \%$, STD) ST positivity by abstracting the RLU values from hard copy records.
Results Per cent CT AT positive declined significantly $(p<0.0001)$ from $96.3 \%$ for specimens from clinics with $>10.0 \%$ ST positivity to $90.9 \%$ for specimens from a single clinic with $<6 \%$ ST positivity (see Abstract P3-S1.11 table 1). GC ST positivity was $<6 \%$ for 109 (87\%) of the clinics. In spite of the lower GC ST positivity, the per cent GC AT positive was also $>90 \%$, ranging from $95.4 \%$ for GC ST positivity $<2 \%$ to $97.7 \%$ for GC ST positivity $3.0 \%-4.0 \%$. However, the per cent GC AT positive was not associated with GC ST positivity $(p=0.17)$. Discordant GC AT results were confined to GC ST positive specimens with RLU $<1$ million (results not shown). The per cent of ST specimens with RLU $<1$ million and the per cent AT negative among these lower RLU positives were also not associated with clinic ST positivity ( $\mathrm{p}=0.14$ and $\mathrm{p}=0.78$, respectively).

Abstract P3-S1.11 Table 1 APTIMA additional test results among women by clinic Combo 2 positivity and organism Mississippi-2007

\begin{tabular}{|c|c|c|c|c|c|c|}
\hline \multirow[b]{3}{*}{ Organism } & \multirow{3}{*}{$\begin{array}{l}\text { Clinic Combo } \\
2 \% \text { positivity }\end{array}$} & \multicolumn{4}{|c|}{ APTIMA AT result } & \multirow[b]{3}{*}{$\mathrm{p}$ Value } \\
\hline & & \multirow[b]{2}{*}{ Retested \# } & \multicolumn{3}{|c|}{ Positive } & \\
\hline & & & \# & $\%$ & $95 \% \mathrm{CI}$ & \\
\hline \multirow[t]{5}{*}{ Ст } & $<6.0$ & 11 & 10 & 90.9 & 58.7 to 99.8 & \multirow[t]{5}{*}{$<0.0001^{*}$} \\
\hline & $6.0-<8.0$ & 281 & 261 & 92.9 & 89.2 to 95.6 & \\
\hline & $8.0-<10.0$ & 799 & 745 & 93.2 & 91.3 to 94.9 & \\
\hline & $10.0+$ & 5462 & 5262 & 96.3 & 95.8 to 96.8 & \\
\hline & Total & 6553 & 6278 & 95.8 & 95.3 to 96.3 & \\
\hline \multirow[t]{6}{*}{ NG } & $<2.0$ & 196 & 187 & 95.4 & 91.5 to 97.9 & \multirow[t]{6}{*}{$0.17^{*}$} \\
\hline & $2.0-<3.0$ & 459 & 442 & 96.3 & 94.1 to 97.8 & \\
\hline & $3.0-<4.0$ & 392 & 383 & 97.7 & 95.7 to 98.9 & \\
\hline & $4.0-<6.0$ & 177 & 171 & 96.6 & 92.8 to 98.8 & \\
\hline & $6.0+$ & 617 & 599 & 97.1 & 95.4 to 98.3 & \\
\hline & Total & 1841 & 1782 & 96.8 & 95.9 to 97.6 & \\
\hline
\end{tabular}

${ }^{*}$ Cochran-Armitage trend test.

Conclusions Performing APTIMA CT or GC ATs added little to Combo 2 ST PPV, although the decrease in per cent AT positive with decreasing ST positivity observed in this study raises concern about Combo 2 PPV at CT prevalence levels lower than $6 \%$. The lack of impact of GC prevalence on GC ST RLU or AT results is unexpected and might indicate that the Combo 2 ST PPV is very high even at the lower GC prevalence. In other words, most negative GC AT results are false rather than true negatives and the patients should be treated.

\section{P3-S1.12 HIGH CONCORDANCE OF TEST RESULTS OF THE CHLAMYDIA TRACHOMATIS DETECTION AND GENOTYPING KIT COMPARED TO THE COBAS AMPLICOR CT/NG TEST}

doi:10.1136/sextrans-2011-050108.412

${ }^{1} \mathrm{~L}$ van Dommelen, ${ }^{2} \mathrm{~A}$ A T P Brink, ${ }^{2} \mathrm{~F} \mathrm{H}$ van Tiel, ${ }^{3} \mathrm{~W} \mathrm{G} V$ Quint, ${ }^{4} \mathrm{~S}$ A Morré, ${ }^{2} \mathrm{P} F$ Wolffs, ${ }^{5} \mathrm{C} C \mathrm{~J}$ P A Hoebe. ${ }^{1} \mathrm{PAMM}$, Veldhoven, Netherlands; ${ }^{2}$ Maastricht University Medical Centre, Netherlands; ${ }^{3} D D L$ Diagnostic Laboratory, Netherlands; ${ }^{4}$ VU University Medical Center, Netherlands; ${ }^{5}$ South Limburg Public Health Service, Netherlands

Background Improving diagnostic methods for the detection of Chlamydia trachomatis (CT), including genotyping, can contribute to control of CT by acquiring knowledge on epidemiology, transmission, sexual networks and pathogenicity. In the present study, we have compared the performance of the Chlamydia trachomatis detection and genotyping (Ct-DT) kit (Labo Bio-medical Products BV, Rijswijk, The Netherlands) with the COBAS Amplicor CT/NG (Roche Diagnostics Systems, Basel, Switzerland) in a well described female population consulting a sexually transmitted infection (STI) clinic.

Methods Self obtained vaginal swabs (SVS) were collected from females visiting a STI clinic. The presence of Chlamydia trachomatis 